\section{Adaptive Coding and Prediction of Sources With Large and Infinite Alphabets}

Boris Y. Ryabko, Member, IEEE, Jaakko Astola, Fellow, IEEE, and Alex Gammerman

\begin{abstract}
The problem of predicting a sequence $x_{1}, x_{2}, \ldots$ generated by a discrete source with unknown statistics is considered. Each letter $x_{t+1}$ is predicted using the information on the word $x_{1} x_{2} \cdots x_{t}$ only. This problem is of great importance for data compression, because of its use to estimate probability distributions for PPM algorithms and other adaptive codes. On the other hand, such prediction is a classical problem which has received much attention. Its history can be traced back to Laplace. We address the problem where the sequence is generated by an independent and identically distributed (i.i.d.) source with some large (or even infinite) alphabet and suggest a class of new methods of prediction.
\end{abstract}

Index Terms-Adaptive coding, Laplace problem of succession, lossless data compression, prediction of random processes, Shannon entropy, source coding.

\section{INTRODUCTION}

$\mathbf{T}$ HE problem of prediction can be traced back to Laplace [7], [36]. Presently, the problem of prediction is investigated by many researchers because of its practical applications and importance for probability theory, information theory, statistics and other theoretical sciences, see [1], [15], [16], [18], [22], [24], and [34]-[36].

Consider a source with unknown statistics which generates sequences $x_{1} x_{2} \cdots$ of letters from an alphabet $A=\left\{a_{1}, \ldots, a_{n}\right\}$. The underlying true distribution, which is unknown, is indicated by the letter $p$. Let the source generate a message $x_{1} \ldots x_{t-1} x_{t} \ldots$, $x_{i} \in A$ for all $i$, and let $\nu^{t}(a)$ denote the count of letter $a$ occurring in the word $x_{1} \ldots x_{t-1} x_{t}$. After the first $t$ letters $x_{1}, \ldots, x_{t-1}, x_{t}$ have been processed the following letter $x_{t+1}$ needs to be predicted. By definition, the prediction is the set of nonnegative numbers $p^{*}\left(a_{1} \mid x_{1} \cdots x_{t}\right), \ldots, p^{*}\left(a_{n} \mid x_{1} \cdots x_{t}\right)$ which are estimates of the unknown conditional probabilities $p\left(a_{1} \mid x_{1} \cdots x_{t}\right), \ldots, p\left(a_{n} \mid x_{1} \cdots x_{t}\right)$, i.e., of the probabilities $p\left(x_{t+1}=a_{i} \mid x_{1} \cdots x_{t}\right) ; i=1, \ldots, n$.

Laplace suggested the following prediction $p_{L}^{*}\left(a \mid x_{1} \cdots x_{t}\right)=$ $\left(\nu^{t}(a)+1\right) /(t+|A|)$, where $|A|$ is the number of letters in the alphabet $A,[7]$, [36]. (The problem which Laplace considered was to estimate the probability that the sun will rise tomorrow, given that it has risen every day since the creation. Using our terminology, we can say that Laplace estimated $p(r \mid r r \cdots r)$ and $p(\bar{r} \mid r r \cdots r)$, where $\{r, \bar{r}\}$ is the alphabet ("sun rises", "sun does not rise") and the length of $r r \cdots r$ is the number of days since the creation.)

We will estimate the precision of the prediction by the Kullback-Leibler divergence $\sum_{a \in A} p\left(a \mid x_{1} \cdots x_{t}\right) \log \left(p\left(a \mid x_{1} \cdots x_{t}\right) /\right.$ $\left.p_{\gamma}^{*}\left(a \mid x_{1} \cdots x_{t}\right)\right)$ between the true distribution $p\left(. \mid x_{1} \ldots x_{t}\right)$ and its

Manuscript received October 1, 2005; revised May 5, 2007. This work was supported by the joint project grant "Efficient randomness testing of random and pseudorandom number generators" of Royal Society, U.K. (Grant ref: 15995) and Russian Foundation for Basic Research (Grant 06-07-89025-a).

B. Y. Ryabko is with Siberian State University of Telecommunications and Informatics, and Institute of Computational Technologies of Siberian Branch of Russian Academy of Science, Novosibirsk 630102, Russia (e-mail: boris@ryabko.net).

J. Astola is with the Tampere University of Technology, FIN-33101, Finland (e-mail: jta@cs.tut.fi).

A. Gammerman is with Department of Computer Science, Royal Holloway, University of London, London, U.K. (e-mail: alex@cs.rhul.ac.uk).

Communicated by M. Effros, Associate Editor for Source Coding.

Digital Object Identifier 10.1109/TIT.2008.926385 estimation $p_{\gamma}^{*}\left(. \mid x_{1} \ldots x_{t}\right)$, where $\gamma$ denotes a prediction method (here and below $\left.\log x \equiv \log _{2} x\right)$.

In data compression the average divergence is called redundancy and plays a key rule. Let us comment on the relation to data compression in more detail. An encoder can construct a code with codelength $l^{*}\left(a \mid x_{1} \cdots x_{t}\right) \approx-\log p^{*}\left(a \mid x_{1} \cdots x_{t}\right)$ for any letter $a \in A$ and the approximation may be as accurate as you like, if the arithmetic code is used, see [28], [23]. An ideal encoder would base coding on the true distribution $p$ and not on the prediction $p^{*}$. The difference in performance (the redundancy) measured by the average code length is given by

$$
\begin{aligned}
\sum_{a \in A} p\left(a \mid x_{1} \cdots x_{t}\right)\left(-\log p^{*}\left(a \mid x_{1} \cdots x_{t}\right)\right) & \\
& -\sum_{a \in A} p\left(a \mid x_{1} \cdots x_{t}\right)\left(-\log p\left(a \mid x_{1} \cdots x_{t}\right)\right) \\
= & \sum_{a \in A} p\left(a \mid x_{1} \cdots x_{t}\right) \log \frac{p\left(a \mid x_{1} \cdots x_{t}\right)}{p^{*}\left(a \mid x_{1} \cdots x_{t}\right)} .
\end{aligned}
$$

So, we can see that from a mathematical point of view the prediction and adaptive coding are identical and can, therefore, be investigated together. Note that such a scheme of adaptive (or universal) coding was suggested in [4] and now is called as a PPM algorithm.

It is known that the redundancy of the Laplace predictor is upper bounded by $(|A|-1) \log e /(t+1)$ [32], [33], if the predictor is applied to an independent and identically distributed (i.i.d.) source (Here $e \approx 2.718$ is the Euler number). Krichevsky [20], [21] investigated the problem of optimal minimax predictor for i.i.d. sources and showed that, loosely speaking, the redundancy of the optimal predictor is asymptotically equal to $(|A|-1) \log e /(2 t)+o(1 / t)$.

We can see that, on the one hand, the precision of the predictors essentially depends on the alphabet size $|A|$. On the other hand, there are many applications, where the alphabet size is unknown beforehand and can be upper bounded only. Moreover, quite often such a bound is infinity. That is why the problems of prediction and, especially, adaptive (or universal) coding for large and infinite alphabet sources have been a subject in literature before, see [6], [9], [17], [25]-[27], [36]. In particular, it was shown in [26], [27], and [36] that the problem of prediction and universal coding is deeply connected with a classical problem of estimation of the number of species, see [8] and [11] and the review in [27].

In this correspondence we suggest a scheme of prediction and adaptive coding for a case where a source generates letters from an alphabet with unknown (and even infinite) size. This scheme can be applied along with Laplace, Krichevsky and any other predictors. If the suggested scheme is applied to $s$ - letter source and $s$ is unknown, the redundancy is asymptotically the same as if the predictor is applied to the $(s+1)$-letter source and the alphabet size $(s+1)$ is known beforehand.

When the suggested scheme is applied to an infinite alphabet, the redundancy of the code goes to 0 , if, loosely speaking, the original representation of the alphabet letters has a finite average word length. It will be shown that this condition is necessary for the existence of such predictors. In fact, similar results are known in universal coding. Thus, according to Davisson [5], Kieffer [17], Györfi, Páli, and van der Meulen [12], [13], there is a duality between universal source coding and distribution estimation consistent in Kullback-Leibler divergence error. These results imply that, for infinite alphabet, without any condition on the source distribution it is impossible to have universal source code. On the other hand, there are some necessary and sufficient conditions for existence of universal codes (and distribution estimation) suggested by Kieffer [17] and Barron, Györfi and van der Meulen [2] (see also Kieffer and Yang [19] and He and Yang [14] who showed that any 
collection satisfying Keiffer's condition from [17] can be compressed by grammar codes). In this correspondence, we show that the same, in spirit, results are valid for letterwise coding and prediction.

It is important to note that, first, for infinite alphabet the requirement of knowing a code with finite expected length is not trivial. It is not clear how one would have access to such a code. Second, the resulting per-symbol redundancy, while converging to 0 , is not uniformly bounded. Hence while it is true that for every source, as the length of an encoded sequence grows sufficiently large the per-symbol redundancy diminishes to 0 , it is also true that for every sequence, however large, some sources can have per-symbol redundancy that is arbitrarily high. (So, for example, even after compressing a million symbols, given the results presented, the per-symbol redundancy could be one billion.) This should be contrasted with results in [26] where the per-symbol redundancy of the source pattern is uniformly bounded by $1 / n^{1 / 3}$ for all sources.

We mainly consider a case of prediction for i.i.d. sources, because the predictors for i.i.d. sources are used as a "core" in the PPM scheme and other practically used adaptive codes and the performance of those codes effectively depends on the redundancy of the i.i.d. predictors. But all results can be easily extended to Markov sources, using well-known methods, see, for example, [33].

The outline of the correspondence is as follows. The next section contains some necessary facts and definitions. The sections three and four are devoted to description of the new scheme of prediction and its properties, correspondingly. Applications of the new scheme to sources with unknown and infinite alphabets are described in the fifth section. All proofs are given in the Appendix.

\section{DEFINITIONS AND PRELIMINARIES}

Consider an alphabet $A=\left\{a_{1}, \ldots, a_{n}\right\}$ with $n \geq 2$ letters and denote by $A^{t}$ the set of words $x_{1} \cdots x_{t}$ of length $t$ from $A$. Let $p$ be a source which generates letters from $A$. Formally, $p$ is a probability distribution on the set of words of infinite length or, more simply, $p=$ $\left(p^{t}\right)_{t \geq 1}$ is a consistent set of probabilities over the sets $A^{t} ; t \geq 1$. By $M_{0}(\bar{A})$ we denote the set of Bernoulli (or i.i.d.) sources over $A$.

Denote by $D(\cdot \| \cdot)$ the Kullback-Leibler divergence and consider a source $p$ and a method $\gamma$ of prediction. The redundancy is characterized by the divergence

$$
\begin{aligned}
r_{\gamma, p}\left(x_{1} \cdots x_{t}\right)=D & \left(p\left(\cdot \mid x_{1} \cdots x_{t}\right) \| p_{\gamma}^{*}\left(\cdot \mid x_{1} \cdots x_{t}\right)\right) \\
& =\sum_{a \in A} p\left(a \mid x_{1} \cdots x_{t}\right) \log \frac{p\left(a \mid x_{1} \cdots x_{t}\right)}{p_{\gamma}^{*}\left(a \mid x_{1} \cdots x_{t}\right)} .
\end{aligned}
$$

For fixed $t, r_{\gamma, p}$ is a random variable, because $x_{1}, x_{2}, \ldots, x_{t}$ are random variables. We define the average redundancy at time $t$ by

$$
r^{t}(p \| \gamma)=E\left(r_{\gamma, p}(\cdot)\right)=\sum_{x_{1} \cdots x_{t} \in A^{t}} p\left(x_{1} \cdots x_{t}\right) r_{\gamma, p}\left(x_{1} \cdots x_{t}\right) .
$$

Related to this quantity we define the maximum average divergence (at time $t$ ) by

$$
r^{t}(M \| \gamma)=\sup _{p \in M} r^{t}(p \| \gamma)
$$

where $M$ is a set of sources. If the Laplace predictor

$$
p_{L}^{*}\left(a \mid x_{1} \cdots x_{t}\right)=\left(\nu^{t}(a)+1\right) /(t+|A|)
$$

is applied to the set of i.i.d. sources $M_{0}(A)$, its average redundancy $\left(r^{t}(p \| L)\right)$ is upper bounded by $(|A|-1) \log e /(t+1)$, i.e.

$$
r^{t}\left(M_{0} \| L\right) \leq(|A|-1) \log e /(t+1),
$$

see [32], [33] (as before, $\nu^{t}(a)$ denotes the count of the letter $a$ occurring in the word $\left.x_{1} \ldots x_{t-1} x_{t}\right)$. In [21], Krichevsky investigated the problem of optimal predictor for the set of i.i.d. sources and showed that for any predictor $\pi$ the maximal redundancy is asymptotically lower bounded by $(|A|-1) \log e / 2 t$

$$
\lim _{t \rightarrow \infty} \sup \left(2 t r^{t}\left(M_{0} \| \pi\right)\right) \geq(|A|-1) \log e .
$$

He has also suggested the predictor

$$
p_{K}^{*}\left(a \mid x_{1} \cdots x_{t}\right)=\left(\nu^{t}(a)+\delta\right) /(t+\delta|A|)
$$

where $\delta=0.50922 \ldots$, and shown that it is asymptotically optimal

$$
\lim _{t \rightarrow \infty} \sup \left(2 t r^{t}\left(M_{0} \| K_{1}\right)\right)=(|A|-1) \log e .
$$

As we mentioned above, predictors for i.i.d. sources can be easily extended to Markov sources (see, for example, [20], [33]) and to the general stationary and ergodic sources, as it was suggested in [30], [31]. But, it is worth noting that, as it is shown in [31], there exist such stationary and ergodic sources that their divergence does not go to 0 . More precisely, for any predictor $\gamma$ there exists such a stationary and ergodic source $\tilde{p}$, that $\lim _{t \rightarrow \infty} \sup \left(r^{t}(\tilde{p} \| \gamma)\right) \geq 1$, (see [31], [1], and [22]). But, on the other hand, it is shown in [30], [31] that there exists such a predictor $\rho$, that the average $R^{t}(p \| \rho)=t^{-1} \sum_{i=1}^{t} r^{i}(p \| \rho)$ goes to 0 for any stationary and ergodic source $p$, where $t$ goes to infinity. We will focus our attention on the per letter redundancy $r^{t}(\|)$ ), but all estimates can be easily extended to $R^{t}(\|)$.

\section{THE NEW SCHEME}

Let, as before, $p$ be an i.i.d. source generating letters from the alphabet $A$. The probability distribution $p(a), a \in A$ is unknown and each letter $x_{t+1}$ should be predicted (or encoded) using information on the word $x_{1} x_{2} \cdots x_{t}$ only.

The suggested scheme can be applied to any predictor (or letterwise coding), but we will use the Laplace predictor as the main example. We start the description of the new scheme using a simple example. Let $A=\left\{a_{0}, a_{1}, a_{2}\right\}$ and $t=4, x_{1} x_{2} x_{3} x_{4}=a_{0} a_{2} a_{0} a_{0}$. The Laplace predictor is as follows:

$$
\begin{aligned}
& p_{L}^{*}\left(x_{5}=a_{0}\right)=(3+1) /(4+3)=4 / 7 \\
& p_{L}^{*}\left(x_{5}=a_{1}\right)=(0+1) /(4+3)=1 / 7 \\
& p_{L}^{*}\left(x_{5}=a_{2}\right)=(1+1) /(4+3)=2 / 7
\end{aligned}
$$

see (4). In this example, we suggest to group letters into two subsets $A_{0}=\left\{a_{0}, a_{1}\right\}, A_{1}=\left\{a_{2}\right\}$ and carry out the prediction into two steps. First, the original sequence $a_{0} a_{2} a_{0} a_{0}$ is represented as $A_{0} A_{1} A_{0} A_{0}$ and the membership in the subsets is predicted as follows:

$$
\begin{aligned}
& p_{L}^{*}\left(x_{5} \in A_{0}\right)=(3+1) /(4+2)=2 / 3 \\
& p_{L}^{*}\left(x_{5} \in A_{1}\right)=(1+1) /(4+2)=1 / 3 .
\end{aligned}
$$

We know that $A_{1}$ contains one letter $\left(a_{2}\right)$, hence, $p_{L}^{*}\left(x_{5}=a_{2}\right)=$ $1 / 3$. The sequence $A_{0} A_{1} A_{0} A_{0}$, which contains three letters $A_{0}$, is used for predicting conditional probabilities $p\left(x_{5}=a_{i} / x_{5} \in A_{0}\right)$, $i=0,1$. Again, we apply the Laplace predictor (4) to the sequence $A_{0} A_{0} A_{0}=a_{0} a_{0} a_{0}$ and obtain the following prediction: $p_{L}^{*}\left(x_{5}=\right.$ $\left.a_{0} / x_{5} \in A_{0}\right)=(3+1) /(3+2)=4 / 5, p_{L}^{*}\left(x_{5}=a_{1} / x_{5} \in A_{0}\right)=$ $(0+1) /(3+2)=1 / 5$. So, combining all predictions, we obtain $p_{L}^{*}\left(x_{5}=a_{0}\right)=(2 / 3)(4 / 5)=8 / 15, p_{L}^{*}\left(x_{5}=a_{1}\right)=(2 / 3)(1 / 5)=$ $2 / 15, p_{L}^{*}\left(x_{5}=a_{2}\right)=1 / 3$. We can see that this new prediction and the Laplace predictor (4) are different. It is worth noting that this partitioning trick can be used in case of non discrete alphabet, see [2]. 


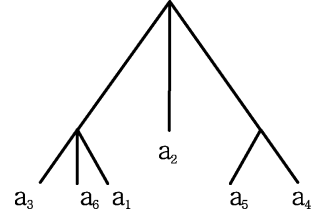

Fig. 1. $A_{\alpha_{1}}=\left\{a_{1}, a_{3}, a_{6}\right\}, A_{\alpha_{2}}=\left\{a_{2}\right\}, A_{\alpha_{3}}=\left\{a_{4}, a_{5}\right\}, A_{\alpha_{1,1}}=\left\{a_{3}\right\}, \ldots$

It will be convenient to describe the general case using the notation of a tree. Let $\Upsilon$ be a rooted tree, which contains $|A|$ leaves, and let each leaf be marked by one letter from $A$ in such a way that different leaves are marked by different letters.

We will mark each vertex $\mu \in \Upsilon$ by a subset $A_{\mu}$ as follows. We consider the subtree $\Upsilon_{\mu}$ whose root is the vertex $\mu$ and define the subset $A_{\mu}$ by the set of all letters, which mark the leaves of the subtree $\Upsilon_{\mu}$. Note that $\Upsilon_{\text {root }}=A$. We denote vertices of depth one by $\alpha_{i}, i=1, \ldots, k$, the vertices of the depth 2 by $\alpha_{i, j}, j=1, \ldots, k_{i}, i=1, \ldots, k$, etc., where $k$ is the number of the depth 1 vertices, $k_{i}$ is the number of the sons of the vertex $i$, etc. The prediction is carried out as follows. First a generated sequence $x_{1} \ldots x_{t}$ is represented as the sequence $A_{\alpha_{i_{1}}} A_{\alpha_{i_{2}}} \ldots A_{\alpha_{i_{t}}}$, where $\alpha_{i_{j}}$ is such a vertex of depth one that the letter $x_{j}$ belongs to the subset $A_{\alpha_{i j}}$. Then, $A_{\alpha_{i_{1}}} A_{\alpha_{i_{2}}} \ldots A_{\alpha_{i_{t}}}$ is considered as the sequence generated by an i.i.d. source with the alphabet $\left\{A_{\alpha_{i}}, i=1, \ldots, k\right\}$ and the next letter $A_{\alpha_{i}+1}$ is predicted (say, by Laplace predictor). In fact, $p_{L}^{*}\left(A_{\alpha_{i}}\right)$ is the estimation of $p\left(x_{t+1} \in\right.$ $A_{\alpha_{i}}$ ). Then, for each vertex $\alpha_{i, j}, i=1, \ldots, k, j=1, \ldots, k_{i}$ of the depth 2, which is not a leaf, we estimate the probability $p\left(x_{t+1} \in\right.$ $\left.A_{\alpha_{i, j}} / x_{t+1} \in A_{\alpha_{i}}\right)$. For the purpose for each $i$, we consider all letters $A_{\alpha_{i}}$ in the sequence $A_{\alpha_{i_{1}}} A_{\alpha_{i_{2}}} \ldots A_{\alpha_{i_{t}}}$ and organize the following sequence of their sons $A_{\alpha_{i, j_{1}}} \ldots A_{\alpha_{i, j_{s}}}$, whose length $s$ equals the count of occurrences $A_{\alpha_{i}}$ in the sequence $A_{\alpha_{i_{1}}} A_{\alpha_{i_{2}}} \ldots A_{\alpha_{i_{t}}}$. This sequence is considered as a generated by an i.i.d. source and the next letter $A_{\alpha_{i, j} j_{s}}$ is predicted. As a result, we obtain $p_{L}^{*}\left(A_{\alpha_{i, j}}\right)$, which are, in fact, the estimations $p_{L}^{*}\left(x_{t+1} \in A_{\alpha_{i, j}} / x_{t+1} \in A_{\alpha_{i}}\right)$, and so on. Then we calculate the predictor $p_{L}^{*}\left(x_{t+1}=a\right)$ as a product of $p_{L}^{*}\left(x_{t+1} \in\right.$ $\left.A_{\alpha_{i}}\right) p_{L}^{*}\left(x_{t+1} \in A_{\alpha_{i, j}} / x_{t+1} \in A_{\alpha_{i}}\right) p_{L}^{*}\left(x_{t+1} \in A_{\alpha_{i, j, m}} / x_{t+1} \in\right.$ $A_{\alpha_{i, j}}$ ) ... where $a \in A_{\alpha_{i}}, a \in A_{\alpha_{i, j}}, a \in A_{\alpha_{i, j, m}}, \ldots$.

The following example is to illustrate all steps. Let the alphabet $A$ be $\left\{a_{1}, \ldots, a_{6}\right\}$ and the tree $\Upsilon$ is shown on the Fig. 1 . Let the generated sequence $x_{1} \ldots x_{t}$ be $a_{3} a_{1} a_{5} a_{5} a_{2} a_{5} a_{4} a_{2} a_{3}$. According to the tree $\Upsilon$, firstly this sequence is represented as $A_{\alpha_{1}} A_{\alpha_{1}} A_{\alpha_{3}} A_{\alpha_{3}} A_{\alpha_{2}} A_{\alpha_{3}} A_{\alpha_{3}} A_{\alpha_{2}} A_{\alpha_{1}}$ and the next letter $x_{t+1}$ is predicted as follows: $p_{L_{\Upsilon}}^{*}\left(x_{t+1} \in A_{\alpha_{1}}\right)=(3+1) /(9+3)$, $p_{L_{\Upsilon}}^{*}\left(x_{t+1} \in A_{\alpha_{2}}\right)=(2+1) /(9+3), p_{L_{\Upsilon}}^{*}\left(x_{t+1} \in A_{\alpha_{3}}\right)=$ $(4+1) /(9+3)$. Then we divide the sequence of subsets $A_{\alpha_{1}} A_{\alpha_{1}} A_{\alpha_{3}} A_{\alpha_{3}} A_{\alpha_{2}} A_{\alpha_{3}} A_{\alpha_{3}} A_{\alpha_{2}} A_{\alpha_{1}}$ into the three following subsequences $A_{\alpha_{1}} A_{\alpha_{1}} A_{\alpha_{1}}, \quad A_{\alpha_{2}} A_{\alpha_{2}}$ and $A_{\alpha_{3}} A_{\alpha_{3}} A_{\alpha_{3}} A_{\alpha_{3}}$. The set $A_{\alpha_{2}}$ contains only one letter $a_{2}$, hence, the prediction of this letter coincides with the prediction of the subset $A_{\alpha_{2}}$ : $p_{L_{\Upsilon}}^{*}\left(x_{t+1}=a_{2}\right)=p_{L_{\Upsilon}}^{*}\left(x_{t+1} \in A_{\alpha_{2}}\right)=(2+1) /(9+3)$. The first subsequence $A_{\alpha_{1}} A_{\alpha_{1}} A_{\alpha_{1}}$ is represented as $a_{3} a_{1} a_{3}$ and the next letter is predicted by $p_{L_{\Upsilon}}^{*}\left(x_{t+1}=a_{1} / x_{t+1} \in A_{\alpha_{1}}\right)=(1+1) /(3+3)$, $p_{L_{\Upsilon}}^{*}\left(x_{t+1}=a_{3} / x_{t+1} \in A_{\alpha_{1}}\right)=(2+1) /(3+3), p_{L_{\Upsilon}}^{*}\left(x_{t+1}=\right.$ $\left.a_{6} / x_{t+1} \in A_{\alpha_{1}}\right)=(0+1) /(3+3)$. The third subsequence $A_{\alpha_{3}} A_{\alpha_{3}} A_{\alpha_{3}} A_{\alpha_{3}}$ is represented as $a_{5} a_{5} a_{5} a_{4}$ and we obtain the following prediction: $p_{L_{\Upsilon}}^{*}\left(x_{t+1}=a_{4} / x_{t+1} \in A_{\alpha_{3}}\right)=(1+1) /(4+2)$, $p_{L_{\Upsilon}}^{*}\left(x_{t+1}=a_{5} / x_{t+1} \in A_{\alpha_{3}}\right)=(3+1) /(4+2)$. Finally, we obtain $p_{L_{\Upsilon}}^{*}\left(x_{t+1}=a_{1}\right)=(4 / 12)(2 / 6), p_{L_{\Upsilon}}^{*}\left(x_{t+1}=a_{2}\right)=(3 / 12)$, $p_{L_{\Upsilon}}^{*}\left(x_{t+1}=a_{3}\right)=(4 / 12)(3 / 6), p_{L_{\Upsilon}}^{*}\left(x_{t+1}=a_{4}\right)=(5 / 12)(2 / 6)$, $p_{L_{\Upsilon}}^{*}\left(x_{t+1}=a_{5}\right)=(5 / 12)(4 / 6), p_{L_{\Upsilon}}^{*}\left(x_{t+1}=a_{6}\right)=(4 / 12)(1 / 6)$. The properties of the suggested scheme are as follows.
Theorem 1: Let $x_{1} \ldots x_{t}$ be a sequence generated by an i.i.d. source from an alphabet $A$. If the letter $x_{t+1}$ is predicted by the suggested scheme according to a tree $\Upsilon$ with the set of vertexes $\Lambda$, then the following upper bound for the precision (or redundancy) (2) is valid:

$$
r^{t}\left(p \| L_{\Upsilon}\right) \leq \log e \sum_{\lambda \in \Lambda}(\sigma(\lambda)-1) \min \left\{1 /(t+1), p\left(A_{\lambda}\right)\right\},
$$

where $\sigma(\lambda)$ is the number of sons of the vertex $\lambda, p\left(A_{\lambda}\right)=$ $\sum_{a \in A_{\lambda}} p(a), L_{\Upsilon}$ is a notation of the predictor.

Proof: As shown in the Appendix.

Comment: The summands, which correspond to leaves in (8), are equal to 0 , because $\sigma(\lambda)=1$, if $\lambda$ is a leaf. So, if we define the set of internal vertexes by $\Lambda_{\text {int }}$ we can rewrite (8) as follows:

$$
r^{t}\left(p \| L_{\Upsilon}\right) \leq \log e \sum_{\lambda \in \Lambda_{\mathrm{int}}}(\sigma(\lambda)-1) \min \left\{1 /(t+1), p\left(A_{\lambda}\right)\right\} .
$$

Corollary: The Laplace predictor (4) corresponds to the tree which consists of a root with $|A|$ sons (leaves). From Theorem 1 we immediately obtain the upper bound (5).

\section{UNKNOWN AND INFINITE ALPHABET}

The suggested scheme can be generalized in such a way that the tree $\Upsilon$, which determines the algorithm of prediction, depends on the encoded part $x_{1} \ldots x_{t}$. We consider one such scheme, which is close in spirit to some methods based on the estimate of an escape probability [36], and show how Theorem 1 can be used for obtaining an asymptotic estimations of the redundancy in the case where the algorithm of prediction (or the tree $\Upsilon$ ) depends on the sequence.

In order to describe this predictor, which is used along with the Laplace method (4), we define $A_{0}^{t}$ as the subset containing all 0-frequent letters at the moment $t$ and let $A_{+}^{t}=A-A_{0}^{t}$. The predictor $\eta_{t}$ is defined as follows:

$$
\eta^{t}(a)= \begin{cases}\left(\nu^{t}(a)+1\right) /\left(t+\left|A_{+}^{t}\right|+1\right), & \text { if } \nu^{t}(a)>0 \\ 1 /\left(\left(t+\left|A_{+}^{t}\right|+1\right)\left|A_{0}^{t}\right|\right), & \text { if } \nu^{t}(a)=0\end{cases}
$$

In words, the letter $x_{t+1}$ is encoded according to the tree $\Upsilon_{t}$, whose root has $\left|A_{+}^{t}\right|+1$ sons. Of these, $\left|A_{+}^{t}\right|$ are leaves corresponding to the letters from the set $A_{+}^{t}$ (i.e., their frequencies are greater than 0 ). One son of the root corresponds to the set $A_{0}^{t}$ and has $\left|A_{0}^{t}\right|$ sons, which, in turn, correspond to the letters from $A_{0}^{t}$.

This code is related to some methods from [36], which are based on estimate of the escape probability. It is interesting that, asymptotically, the increase of the redundancy is the same as if the alphabet size would increase by one. In words, if the suggested scheme is applied to $s$-letter source and $s$ is unknown, the redundancy is the same as if the predictor is applied to $(s+1)$ - letter source and the alphabet size $(s+1)$ is known beforehand. More precisely, the following property is valid.

Theorem 2: Let the predictor $\eta_{t}$ be applied to an i.i.d. source $p$ with an alphabet $A$. Then, the following inequality is valid:

$$
\limsup _{t \rightarrow \infty}\left(t r^{t}\left(p \| \eta_{t}\right)\right) \leq \log e \min \{s,|A|-1\}
$$

where $s$ is the number of the alphabet letters whose probability is not 0 .

Proof: As shown in the Appendix.

Comment: The Krichevsky predictor (6) can be used along with the described scheme (instead of the Laplace predictor). If we define this predictor as $\tilde{\eta}_{t}$, then the following inequality is valid:

$$
\limsup _{t \rightarrow \infty}\left(2 t r^{t}\left(p \| \tilde{\eta}_{t}\right)\right) \leq \log e \min \{s,|A|-1\} .
$$


If we compare these inequalities with the redundancy of the common Laplace predictor (5) and the Krichevsky one (7), we can see that the "payment" for the lack of knowledge of the alphabet size is asymptotically as large as it could be caused by increasing the alphabet by one letter. In other words, if the letters with nonzero probabilities are known beforehand, the redundancy of the common Laplace predictor is $\log e(s-1) /(t+1)$, where $s$ is the number of letters with nonzero probabilities.

Now we consider the case of infinite alphabet $A=$ $\left\{a_{1}, \ldots, a_{n}, \ldots\right\}$. We suppose that there is an i.i.d. source, which generates letters from $A$ with a probability distribution $p$. Obviously, the first letter $x_{1}$ must be encoded by an encoder and decoded by a decoder based on an initial code (over some finite alphabet $B$, say, $B=\{0,1\})$ and this code is known to both the encoder and the decoder. (Otherwise, the first letter cannot be transmitted.) We denote this initial code by $c=\left\{c_{1}, c_{2}, \ldots\right\}$ and suppose that the letter $a_{i}$ is encoded by the word $c_{i}$. Of course, we also suppose that the code $c$ is uniquely decodable. Moreover, we will suppose that $c$ is the prefix code, because it is known that for each decodable code $c$ there exists such a prefix code $c^{*}$ that their codeword lengths are equal $\left(\left|c_{i}\right|=\left|c_{i}^{*}\right|\right.$ for all $i$ ), see [10].

If one wants to encode and decode the first letter $x_{1}$, the average codeword length of this letter should be finite, i.e.

$$
E_{p}\left(c_{i}\right) \equiv \sum_{i=1}^{\infty} p\left(a_{i}\right)\left|c_{i}\right|<\infty .
$$

For example, if the (binary) code and the probability distribution are as follows:

$$
\begin{aligned}
c_{1} & =0, c_{2}=10, c_{3}=110, c_{4}=1110, \cdots \\
p\left(a_{1}\right) & =1 / 2, p\left(a_{2}\right)=1 / 4, p\left(a_{3}\right)=1 / 8, \cdots
\end{aligned}
$$

then the average codeword length $E_{p}\left(\left|c_{i}\right|\right)=2$ bits and, hence, such a code can be used for encoding $x_{1}$. On the other hand, consider the same probability distribution and a new code $\bar{c}$ such that $\left|\bar{c}_{i}\right|=2^{i}$. This code cannot be used, because its average codeword length is infinite and the first letter $x_{1}$ cannot be transmitted from an encoder to a decoder. So, our requirement is as follows. We do not know the source probabilities, but know a code $c$, whose average codeword length is finite. The following theorem shows that this requirement is sufficient for the existence of such adaptive code whose redundancy goes to 0 . In a certain sense, this requirement is necessary, because one cannot transmit the first letter of the generated sequence if she/he does not have such an initial code.

Theorem 3: Suppose that there are a source generated letters from an infinite alphabet $A$ with (unknown) distribution $p$ and a prefix code $c(a), a \in A$, over some finite alphabet such that the average length $E_{p}(|c(a)|)$ is finite. Denote by $\Gamma$ the tree which corresponds to the code $c$ and let $L_{\Gamma}$ be the Laplace predictor corresponding to the tree $\Gamma$. Then the redundancy of this method goes to 0

$$
\lim _{t \rightarrow \infty} r^{t}\left(p \| L_{\Gamma}\right)=0 .
$$

Proof: As shown in the Appendix.

Corollary: If the source alphabet $A$ is infinite, but it is known that only a finite number of letters have nonzero probabilities, the average codeword length (9) is finite for any code and, hence, Theorem 3 is valid for any prefix code.

\section{APPENDIX}

PROOFS

Proof of the Theorem 1: We will use two following lemmas.

Lemma 1: Let a Bernoulli source generate $t$ letters from the alphabet $A^{*}=\{A, \bar{A}\}$ with probabilities $p(A)$ and $p(\bar{A})$ and let $\vartheta(A)$ be a count of occurrence of the letter $A$ in the generated sequence. Then the following inequality is valid:

$$
p(A) E_{p}(1 /(\vartheta(A)+1)) \leq \min \{p(A), 1 /(t+1)\}
$$

where $E_{p}$ is an expectation.

Proof of the Lemma 1: The inequality $p(A) E_{p}(1 /(\vartheta(A)+1)) \leq$ $p(A)$ can be derived from the obvious inequality $E_{p}(1 /(\vartheta(A)+1)) \leq$ $E_{p}(1)=1$

The second inequality $p(A) E_{p}(1 /(\vartheta(A)+1)) \leq 1 /(t+1)$ can be proven as follows:

$$
\begin{aligned}
p(A) & E_{p}(1 /(\vartheta(A)+1) \\
& \left.=p(A) \sum_{j=0}^{t}\left(\begin{array}{l}
t \\
j
\end{array}\right) p(A)^{j}(1-p(A))^{t-j} /(j+1)\right) \\
& \left.\leq \frac{1}{t+1} \sum_{s=0}^{t+1}\left(\begin{array}{c}
t+1 \\
s
\end{array}\right) p(A)^{s}(1-p(A))^{t+1-s}\right)=\frac{1}{t+1} .
\end{aligned}
$$

The lemma is proven.

Lemma 2: Let a Bernoulli source generate $t$ letters from the finite alphabet $A=\{a\}$ with probabilities $p(a), a \in A$. Then the redundancy of the Laplace predictor (4) can be upper bounded by $\log e(|A|-$ 1) $/(t+1)$.

(Although Lemma 2 is known for the Laplace predictor ([32], [33]) we give the details of the proof for the convenience of the reader.)

We employ the general inequality

$$
D(\mu \| \eta) \leq \log e\left(-1+\sum_{a \in A} \mu(a)^{2} / \eta(a)\right)
$$

valid for any distributions $\mu$ and $\eta$ over $A$ (follows from the elementary inequality $\ln x \leq x-1$ ). From the definition of the redundancy (or precision) (1), (2) and (4), we obtain

$$
\begin{aligned}
r^{t} & (p \| L) \\
& =E_{p^{t}} D\left(p\left(\cdot \mid x_{1} \cdots x_{t}\right) \| p^{*}\left(\cdot \mid x_{1} \cdots x_{t}\right)\right) \\
& =E_{p^{t}}\left(D\left(p \| p_{L}^{*}\left(\cdot \mid x_{1} \cdots x_{t}\right)\right)\right. \\
& \leq \log e\left(-1+\sum_{x_{1} \cdots x_{t} \in A^{t}} p\left(x_{1} \cdots x_{t}\right) \sum_{a \in A} \frac{p(a)^{2}(t+|A|)}{\nu_{a}\left(x_{1} \cdots x_{t}\right)+1}\right) \\
& =\log e\left(-1+\sum_{a \in A i=0} \sum^{t} \frac{p(a)^{2}(t+|A|)}{i+1}\left(\begin{array}{c}
t \\
i
\end{array}\right) p(a)^{i}(1-p(a))^{t-i}\right) \\
& =\log e\left(-1+\frac{t+|A|}{t+1} \sum_{a \in A} p(a) \sum_{i=0}^{t}\left(\begin{array}{c}
t+1 \\
i+1
\end{array}\right)\right. \\
\left.p(a)^{i+1}(1-p(a))^{t-i}\right) & \leq \log e\left(-1+\frac{t+|A|}{t+1} \sum_{a \in A} p(a)\right. \\
& \left.\sum_{j=0}^{t+1}\left(\begin{array}{c}
t+1 \\
j
\end{array}\right) p(a)^{j}(1-p(a))^{t+1-j}\right) \\
& =\log e \frac{|A|-1}{t+1} .
\end{aligned}
$$

The lemma is proven.

Consider a letter $a \in A$ and let $\alpha_{i_{1}}, \alpha_{i_{1}, i_{2}}, \ldots, \alpha_{i_{1}, i_{2}, \ldots, i_{s}}$ be a sequence of vertexes such that

$$
A_{\alpha_{i_{1}}} \supset A_{\alpha_{i_{1}, i_{2}}} \cdots \supset A_{\alpha_{i_{1}, i_{2}, \ldots, i_{s}}}
$$


and $A_{\alpha_{i_{1}, i_{2}, \ldots, i_{S}}}=\{a\}$. The estimation of the probability (or prediction) $p\left(x_{t+1}=a\right)$ can be represented as follows:

$$
\begin{aligned}
p_{L_{\Upsilon}}^{*}(a)= & \left(\left(\nu^{t}\left(A_{\alpha_{i_{1}}}\right)+1\right) /(t+\sigma(\text { root }))\right) \\
& \left(\left(\nu^{t}\left(A_{\alpha_{i_{1}, i_{2}}}\right)+1\right) /\left(\nu^{t}\left(A_{\alpha_{i_{1}}}\right)+\sigma\left(\alpha_{i_{1}}\right)\right)\right) \\
& \left(\nu^{t}\left(A_{\alpha_{i_{1}, i_{2}, i_{3}}}\right)+1\right) /\left(\nu^{t}\left(A_{\alpha_{i_{1}, i_{2}}}\right)+\sigma\left(\alpha_{i_{1}, i_{2}}\right)\right) \ldots \\
& \left(\nu^{t}\left(A_{\alpha_{i_{1}, \ldots, i_{s}}}\right)+1\right) /\left(\nu^{t}\left(A_{\alpha_{i_{1}, \ldots, i_{s-1}}}\right)+\sigma\left(\alpha_{i_{1}, \ldots, i_{s-1}}\right)\right)
\end{aligned}
$$

where $\nu^{t}\left(A_{\alpha}\right)$ is a number of occurrences of letters from the subset $A_{\alpha}$ in the sequence $x_{1} \ldots x_{t}$. From this equality and the definition of the redundancy, we obtain the following equality:

$$
\begin{aligned}
r^{t}\left(p \| L_{\Upsilon}\right) & =E_{p\left(x_{1} \ldots x_{t}\right)}\left(\sum_{a \in A} p(a) \log \left(p(a) / p_{L_{\Upsilon}}^{*}(a)\right)\right. \\
= & E\left(\sum _ { a \in A } p ( a ) \left\{\log \frac{p\left(A_{\alpha_{i_{1}}}\right)}{\left(\nu^{t}\left(A_{\alpha_{i_{1}}}\right)+1\right) /(t+\sigma(\text { root }))}\right.\right. \\
& +\log \frac{p\left(A_{\alpha_{i_{1}, i_{2}}}\right) / p\left(A_{\alpha_{i_{1}}}\right)}{\left(\nu^{t}\left(A_{\alpha_{i_{1}, i_{2}}}\right)+1\right) /\left(\nu^{t}\left(A_{\alpha_{i_{1}}}\right)+\sigma\left(\alpha_{i_{1}}\right)\right)}+ \\
& \log \frac{p\left(A_{\alpha_{i_{1}, i_{2}, i_{3}}}\right) / p\left(A_{\alpha_{i_{1}, i_{2}}}\right)}{\left(\nu ^ { t } \left(A_{\left.\left.\alpha_{i_{1}, i_{2}, i_{3}}\right)+1\right) /\left(\nu^{t}\left(A_{\alpha_{i_{1}, i_{2}}}+\sigma\left(\alpha_{i_{1}, i_{2}}\right)\right)\right.}^{p} \cdots+\right.\right.}+ \\
& \left.\left.\log \frac{p\left(A_{\alpha_{i_{1}}, \ldots, i_{s}}\right) / p\left(A_{\alpha_{i_{1}, \ldots, i_{s}-1}}\right)}{\left(\nu^{t}\left(A_{\alpha_{i_{1}, \ldots, i_{s}}}\right)+1\right) /\left(\nu^{t}\left(A_{\alpha_{i_{1}, \ldots, i_{s-1}}}\right)+\sigma\left(\alpha_{i_{1}, \ldots, i_{s-1}}\right)\right)}\right\}\right) .
\end{aligned}
$$

Grouping summands, we obtain

$$
\begin{aligned}
& r^{t}\left(p \| L_{\Upsilon}\right) \\
& =E\left(\sum_{i_{1}} p\left(A_{\alpha_{i_{1}}}\right) \log \frac{p\left(A_{\alpha_{i_{1}}}\right)}{\left(\nu^{t}\left(A_{\alpha_{i_{1}}}\right)+1\right) /(t+\sigma(\text { root }))}\right) \\
& \quad+\sum_{i_{1}} p\left(A_{\alpha_{i_{1}}}\right) E\left(\sum_{i_{2}} \frac{p\left(A_{\alpha_{i_{1}, i_{2}}}\right)}{p\left(A_{\alpha_{i_{1}}}\right)}\right. \\
& \left.\quad \log \frac{p\left(A_{\alpha_{i_{1}, i_{2}}}\right) / p\left(A_{\alpha_{i_{1}}}\right)}{\left(\nu^{t}\left(A_{\alpha_{i_{1}, i_{2}}}\right)+1\right) /\left(\nu^{t}\left(A_{\alpha_{i_{1}}}\right)+\sigma\left(\alpha_{i_{1}}\right)\right.}\right)+\ldots \\
& \quad+\sum_{i_{1}} p\left(A_{\alpha_{i_{1}}}\right) \sum_{i_{2}} \frac{p\left(A_{\alpha_{i_{1}, i_{2}}}\right)}{p\left(A_{\alpha_{i_{1}}}\right)} \ldots E\left(\sum_{i_{s}} \frac{p\left(A_{\alpha_{i_{1}}, \ldots, i_{s}}\right)}{p\left(A_{\alpha_{i_{1}}, \ldots, i_{s-1}}\right)}\right. \\
& \left.\quad \log \frac{p\left(A_{\alpha_{i_{1}}, \ldots, i_{s}}\right) / p\left(A_{\alpha_{i_{1}}, \ldots, i_{s-1}}\right)}{\left(\nu^{t}\left(A_{\alpha_{i_{1}}, \ldots, i_{s}}\right)+1\right) /\left(\nu^{t}\left(A_{\alpha_{i_{1}}, \ldots, i_{s}-1}\right)+\sigma\left(\alpha_{i_{1}, \ldots, i_{s-1}}\right)\right)}\right)+\ldots
\end{aligned}
$$

where $E()$ stands for the expectation $E_{p\left(x_{1} \ldots x_{t}\right)}$ (). It is worth noting that such a grouping is correct for the case of infinite $A$, because all values $E($ ) are nonnegative.

Having taken into account this equality and Lemma 2, we can upper bound the redundancy as follows:

$$
\begin{aligned}
& r^{t}\left(p \| L_{\Upsilon}\right) \\
& \leq \log e\{(\sigma(\text { root })-1) /(t+1) \\
& \quad+E\left(\sum_{i_{1}} p\left(A_{\alpha_{i_{1}}}\right) \frac{\sigma\left(\alpha_{i_{1}}\right)-1}{\left(\nu^{t}\left(A_{\alpha_{i_{1}}}\right)+1\right)}\right)+\ldots \\
& \quad+\sum_{i_{1}} p\left(A_{\alpha_{i_{1}}}\right) \sum_{i_{2}} \frac{p\left(A_{\alpha_{i_{1}, i_{2}}}\right)}{p\left(A_{\alpha_{i_{1}}}\right)} \ldots E\left(\sum_{i_{s-1}} \frac{p\left(A_{\alpha_{i_{1}, \ldots, i_{s-1}}}\right)}{p\left(A_{\alpha_{i_{1}, \ldots, i_{s-2}}}\right)}\right. \\
& \left.\quad \frac{\sigma\left(\alpha_{i_{1}, \ldots, i_{s-1}}\right)-1}{\left(\nu^{t}\left(A_{\alpha_{i_{1}}, \ldots, i_{s-1}}\right)+1\right)}+\cdots\right\} .
\end{aligned}
$$

Let us estimate one summand, say

$$
\begin{aligned}
& \sum_{i_{1}} p\left(A_{\alpha_{i_{1}}}\right) \sum_{i_{2}} \frac{p\left(A_{\alpha_{i_{1}, i_{2}}}\right)}{p\left(A_{\alpha_{i_{1}}}\right)} \\
& \sum_{i_{3}} E\left(\frac{p\left(A_{\alpha_{i_{1}, i_{2}, i_{3}}}\right)}{p\left(A_{\alpha_{i_{1}, i_{2}}}\right)} \frac{\sigma\left(\alpha_{i_{1}, i_{2}, i_{3}}\right)-1}{\left(\nu^{t}\left(A_{\alpha_{i_{1}, i_{2}, i_{3}}}\right)+1\right)}\right) .
\end{aligned}
$$

(General case is obvious). From Lemma 1, we obtain the following inequalities:

$$
\begin{aligned}
& \sum_{i_{1}} p\left(A_{\alpha_{i_{1}}}\right) \sum_{i_{2}} \frac{p\left(A_{\alpha_{i_{1}, i_{2}}}\right)}{p\left(A_{\alpha_{i_{1}}}\right)} \\
& E\left(\sum_{i_{3}} \frac{p\left(A_{\alpha_{i_{1}, i_{2}, i_{3}}}\right)}{p\left(A_{\alpha_{i_{1}, i_{2}}}\right)} \frac{\sigma\left(\alpha_{i_{1}, i_{2}, i_{3}}\right)-1}{\left(\nu^{t}\left(A_{\alpha_{i_{1}, i_{2}, i_{3}}}\right)+1\right)}\right) \\
& =\sum_{i_{1}} p\left(A_{\alpha_{i_{1}}}\right) \sum_{i_{2}} \frac{p\left(A_{\alpha_{i_{1}, i_{2}}}\right)}{p\left(A_{\alpha_{i_{1}}}\right)} \sum_{i_{3}}\left(\sigma\left(\alpha_{i_{1}, i_{2}, i_{3}}\right)-1\right) \\
& E\left(\frac{p\left(A_{\alpha_{i_{1}, i_{2}, i_{3}}}\right)}{p\left(A_{\alpha_{i_{1}, i_{2}}}\right)} \frac{1}{\left(\nu^{t}\left(A_{\alpha_{i_{1}, i_{2}, i_{3}}}\right)+1\right)}\right) \\
& \leq \sum_{i_{1}} p\left(A_{\alpha_{i_{1}}}\right) \sum_{i_{2}} \frac{p\left(A_{\alpha_{i_{1}, i_{2}}}\right)}{p\left(A_{\alpha_{i_{1}}}\right)} \sum_{i_{3}}\left(\sigma\left(\alpha_{i_{1}, i_{2}, i_{3}}\right)-1\right) \\
& E \min \left\{\frac{p\left(A_{\alpha_{i_{1}, i_{2}, i_{3}}}\right)}{p\left(A_{\alpha_{i_{1}, i_{2}}}\right)}, \frac{1}{\left(\nu^{t}\left(A_{\alpha_{i_{1}, i_{2}}}\right)+1\right)}\right\} \\
& =\sum_{i_{1}} p\left(A_{\alpha_{i_{1}}}\right) \sum_{i_{2}} \sum_{i_{3}}\left(\sigma\left(\alpha_{i_{1}, i_{2}, i_{3}}\right)-1\right) \\
& \min \left\{\frac{p\left(A_{\alpha_{i_{1}, i_{2}, i_{3}}}\right)}{p\left(A_{\alpha_{i_{1}}}\right)}, E\left(\frac{p\left(A_{\alpha_{i_{1}, i_{2}}}\right) / p\left(A_{\alpha_{i_{1}}}\right)}{\left(\nu^{t}\left(A_{\alpha_{i_{1}, i_{2}}}\right)+1\right)}\right)\right\} \\
& \leq \sum_{i_{1}} p\left(A_{\alpha_{i_{1}}}\right) \sum_{i_{2}} \sum_{i_{3}}\left(\sigma\left(\alpha_{i_{1}, i_{2}, i_{3}}\right)-1\right) \\
& \min \left\{\frac{p\left(A_{\alpha_{i_{1}, i_{2}, i_{3}}}\right)}{p\left(A_{\alpha_{i_{1}}}\right)}, E\left(\frac{1}{\left(\nu^{t}\left(A_{\alpha_{i_{1}}}\right)+1\right)}\right)\right\} \\
& =\sum_{i_{1}} \sum_{i_{2}} \sum_{i_{3}}\left(\sigma\left(\alpha_{i_{1}, i_{2}, i_{3}}\right)-1\right) \\
& \min \left\{p\left(A_{\alpha_{i_{1}, i_{2}, i_{3}}}\right), E\left(\frac{p\left(A_{\alpha_{i_{1}}}\right)}{\left(\nu^{t}\left(A_{\alpha_{i_{1}}}\right)+1\right)}\right)\right\} \\
& \leq \sum_{i_{1}} \sum_{i_{2}} \sum_{i_{3}}\left(\sigma\left(\alpha_{i_{1}, i_{2}, i_{3}}\right)-1\right) \min \left\{p\left(A_{\alpha_{i_{1}, i_{2}, i_{3}}}\right), \frac{1}{(t+1)}\right\} \text {. }
\end{aligned}
$$

From this estimation and the last upper bound for $r^{t}\left(p \| L_{\Upsilon}\right)$, we obtain the inequality

$$
\begin{aligned}
& r^{t}\left(p \| L_{\Upsilon}\right) \leq \log e\{(\sigma(\text { root })-1) /(t+1))+ \\
& \quad \sum_{i_{1}}\left(\sigma\left(\alpha_{i_{1}}\right)-1\right) \min \left\{p\left(A_{\alpha_{i_{1}}}\right), \frac{1}{(t+1)}\right\}+ \\
& \quad \sum_{i_{1}} \sum_{i_{2}}\left(\sigma\left(\alpha_{i_{1}, i_{2}}\right)-1\right) \min \left\{p\left(A_{\alpha_{i_{1}, i_{2}}}\right), \frac{1}{(t+1)}\right\}+ \\
& \left.\quad \sum_{i_{1}} \sum_{i_{2}} \sum_{i_{3}}\left(\sigma\left(\alpha_{i_{1}, i_{2}, i_{3}}\right)-1\right) \min \left\{p\left(A_{\alpha_{i_{1}, i_{2}, i_{3}}}\right), \frac{1}{(t+1)}\right\}+\cdots\right\} .
\end{aligned}
$$

In fact, the last inequality is (8) and Theorem 1 is proven.

Proof of the Theorem 2: Having taken into account that $t \rightarrow \infty$, we can see that, with probability 1 , there exists such $\bar{t}$ that the set $A_{+}^{\bar{t}}$ contains all letters whose probabilities are larger than 0 and $1 /(\bar{t}+1)$ is less than any nonzero probability. First we consider the case where there is at least 1 letter with zero probability (i.e., $s<|A|$ ). Having taken into account that the root of the tree $\eta_{\bar{t}}$ has $(s+1)$ sons, we obtain from Theorem 1 that $r^{\bar{t}}\left(p \| \eta_{\bar{t}}\right) \leq \log e(s+1) /(t+1)$ for any 
$t \geq \bar{t}$. If all letters have nonzero probabilities (i.e., $s=|A|$ ) then the root of the tree has $|A|$ sons and $r^{\bar{t}}\left(p \| \eta_{\bar{t}}\right) \leq \log e(|A|-1) /(t+1)$. Theorem 2 is proven.

Proof of the Theorem 3: Let $\varepsilon>0$ be some number. There exists such a finite subset $A_{\varepsilon} \subset A$ that

$$
\sum_{a \in\left(A-A_{\varepsilon}\right)} p(a)|C(a)|<\varepsilon /(2 \log e)
$$

because the sum $\sum_{a \in A} p(a)|C(a)|$ is finite. The redundancy of the code $L_{\Gamma}$ can be represented as two following summands:

$$
\begin{aligned}
r^{t}\left(p \| L_{\Gamma}\right)=\sum_{a \in A_{\varepsilon}} p(a) \log \left(p(a) / p_{L_{\Gamma}^{t}}^{*}(a)\right) & \\
& +\sum_{a \in\left(A-A_{\varepsilon}\right)} p(a) \log \left(p(a) / p_{L_{\Gamma}^{t}}^{*}(a)\right),
\end{aligned}
$$

where $L_{\Gamma}^{t}$ is the Laplace probability assignment at the moment $t$. The number of the vertexes in the subtree corresponding to $A_{\varepsilon}$ is finite. Hence, if we apply Theorem 1, we obtain the following upper bound for the first summand in (11):

$$
\sum_{a \in A_{\varepsilon}} p(a) \log \left(p(a) / p_{L_{\Gamma}^{t}}^{*}(a)\right) \leq \text { const } /(t+1) .
$$

Hence, there exists such $t_{0}$ that the first summand in (11) is less than $\varepsilon / 2$, if $t>t_{0}$. Let us estimate the second summand in (11). First we define the subset $\Lambda_{\varepsilon}$ as containing all vertexes which belong to paths from the root to each letter from $A-A_{\varepsilon}$. Applying the Theorem 1, we obtain

$$
\begin{aligned}
\sum_{a \in\left(A-A_{\varepsilon}\right)} p(a) \log \left(p(a) / p_{L_{\Gamma}^{t}}^{*}(a)\right) & \leq \log e \sum_{\lambda \in \Lambda_{\varepsilon}} p\left(A_{\lambda}\right) \\
& =\log e \sum_{\lambda \in \Lambda_{\varepsilon}}\left(\sum_{a \in A_{\lambda}} p(a)\right) .
\end{aligned}
$$

If we define the number of vertex belonged to the path from the root to the leaf $a$ as $\chi(a)$ we can rewrite the last inequality as follows.

$$
\sum_{a \in\left(A-A_{\varepsilon}\right)} p(a) \log \left(p(a) / p_{L_{\Gamma}^{t}}^{*}(a)\right) \leq \log e \sum_{a \in A_{\varepsilon}} p(a) \chi(a) .
$$

By definition, the codeword length $|C(a)|$ of any letter $a$ is equal to $\chi(a)$. Hence, having taken into account this fact, from the latest inequality and (10), we obtain the following upper bound for the second summand:

$$
\sum_{a \in\left(A-A_{\varepsilon}\right)} p(a) \log \left(p(a) / p_{L_{\Gamma}^{t}}^{*}(a)\right) \leq \varepsilon / 2 .
$$

So, each summands in (11) is upper bounded by $\varepsilon / 2$ for any positive $\varepsilon$. Theorem 3 is proven.

\section{REFERENCES}

[1] P. Algoet, "Universal schemes for learning the best nonlinear predictor given the infinite past and side information," IEEE Trans. Inf. Theory, vol. 45, pp. 1165-1185, 1999.

[2] A. R. Barron, L. Györfi, and E. C. van der Meulen, "Distribution estimation consistent in total variation and in two types of information divergence," IEEE Trans. Inf. Theory, vol. 38, pp. 1437-1454, 1992.

[3] P. Billingsley, Ergodic Theory and Information. New York: Wiley, 1965.

[4] J. Cleary and I. Witten, "Data compression using adaptive coding and partial string matching," IEEE Trans. Commun., vol. 32, pp. 396-402, 1984.

[5] L. D. Davisson, "Universal noiseless coding," IEEE Trans. Inf. Theory, vol. 19, pp. 783-795, 1973.

[6] P. Elias, "Universal codeword sets and representations of integers," IEEE Trans. Inf. Theory, vol. 21, pp. 194-203, 1975.
[7] W. Feller, An Introduction to Probabability Theory and Its Applications. New York: John Wiley \& Sons, 1970, vol. 1

[8] R. Fisher, A. Corbet, and C. Williams, "The relation between the number of species and the number of individuals in a random sample of an animal population," J. Animal Ecol., vol. 12, no. 1, pp. 42-48, 1943.

[9] D. P. Foster and R. A. Stine, "Universal codes for finite sequences of integers drawn from a monotone distribution," IEEE Trans. Inform. Theory, vol. 48, pp. 1713-1720, 2002.

[10] R. G. Gallager, Information Theory and Reliable Communication. New York: John Wiley \& Sons, 1968.

[11] I. J. Good and G. H. Toulmin, "The number of new species and the increase in population coverage when the sample is increased," Biometrica, vol. 43, no. 1, pp. 45-63, 1956

[12] L. Györfi, I. Páli, and E. C. van der Meulen, "On universal noiseless source coding for infinite source alphabet," European Trans. Telecommun., vol. 4, pp. 9-16, 1993.

[13] L. Györfi, I. Páli, and E. C. van der Meulen, "There is no universal code for infinite alphabet," IEEE Trans. Inf. Theory, vol. 40, pp. 267-271, 1994.

[14] D. K. He and E. H. Yang, "The universality of grammar- based codes for sources with countablye infinite alphabets," IEEE Trans. Inf. Theory, vol. 51, pp. 3753-3765, 2005.

[15] P. Jacquet, W. Szpankowski, and I. Apostol, "Universal predictor based on pattern matching," IEEE Trans. Inf. Theory, vol. 48, pp. 1462-1472, 2002.

[16] N. Jevtic, A. Orlitsky, and N. P. Santhanam, "A lower bound on compression of unknown alphabets," Theoretic. Comput. Sci., vol. 332, pp. 293-311, 2004.

[17] J. Kieffer, "A unified approach to weak universal source coding," IEEE Trans. Inf. Theory, vol. 24, pp. 674-682, 1978.

[18] J. Kieffer, Prediction and Information Theory 1998 [Online]. Available: ftp://oz.ee.umn.edu/users/kieffer/papers/prediction.pdf/, to be published

[19] J. Kieffer and E. Yang, "Grammar- based codes: A new class of universal lossless source codes," IEEE Trans. Inf. Theory, vol. 46, pp. 737-754, 2000

[20] R. Krichevsky, Universal Compression and Retrival. Dordrecht, Germany: Kluver Academic, 1994.

[21] R. Krichevskii, "Laplace's law of succession and universal encoding," IEEE Trans. Inf. Theory, vol. 44, pp. 296-303, 1998.

[22] G. Morvai, S. J. Yakowitz, and P. H. Algoet, "Weakly convergent nonparametric forecasting of stationary time series," IEEE Trans. Inf. Theory, vol. 43, pp. 483-498, 1997.

[23] A. Moffat, R. Neal, and I. Witten, "Arithmetic coding revisited," $A C M$ Trans. Inf. Syst., vol. 16, no. 3, pp. 256-294, 1998.

[24] A. B. Nobel, "On optimal sequential prediction," IEEE Trans. Inform. Theory, vol. 49, pp. 83-98, 2003.

[25] A. Orlitsky and N. P. Santhanam, "Speaking of infinity," IEEE Trans. Inform. Theory, vol. 50, pp. 2215-2230, 2004.

[26] A. Orlitsky, N. P. Santhanam, and J. Zhang, "Universal compression of memoryless sources over unknown alphabets," IEEE Trans. Inf. Theory, vol. 50, pp. 1469-1481, 2004.

[27] A. Orlitsky, N. P. Santhanam, K. Viswanathan, and J. Zhang, "Convergance of profile based estimators," in Proc. 2005 IEEE Int. Symp. Inf. Theory, Adelaide, Australia, pp. 1843-1847.

[28] J. Rissanen, "Generalized Kraft inequality and arithmetic coding," IBM J. Res. Dev., vol. 20, no. 5, pp. 198-203, 1976.

[29] J. Rissanen, "Universal coding, information, prediction, and estimation," IEEE Trans. Inf. Theory, vol. 30, pp. 629-636, 1984.

[30] B. Y. Ryabko, "Twice-universal coding," Probl. Inf. Transm., vol. 20, no. 3, pp. 173-177, 1984.

[31] B. Y. Ryabko, "Prediction of random sequences and universal coding," Probl. Inf. Transm., vol. 24, no. 2, pp. 87-96, 1988.

[32] B. Y. Ryabko, "A fast adaptive coding algorithm," Probl. Inf. Transm., vol. 26, no. 4, pp. 305-317, 1990.

[33] B. Ryabko and F. Topsoe, "On asymptotically optimal methods of prediction and adaptive coding for Markov sources," J. Complex., vol. 18, no. 1, pp. 224-241, 2002.

[34] S. A. Savari, "A probabilistic approach to some asymptotics in noiseless communication," IEEE Trans. Inf. Theory, vol. 46, pp. 1246-1262, 2000.

[35] W. Szpankowski, Average Case Analysis of Algorithms on Sequences. New York: John Wiley and Sons, 2001.

[36] I. Witten and T. Bell, "The zero-frequancy problem: Estimating the probabilities of novel events in adaptive text compression," IEEE Trans. Inf. Theory, vol. 37, pp. 1085-1094, 1991. 